\title{
USUÁRIOS DE CRACK E REINSERÇÃO SOCIAL: REFLEXÕES SOBRE UM ESTUDO DE CASO
}

LACERDA, Bibiana Lima de. Pós-graduanda, programa em Saúde Coletiva do Centro Universitário Metodista do IPA.

PALMEIRO, Paolo Gomes. Mestrando, Programa de Pós-graduação em Biociências e Reabilitação, Centro Universitário Metodista do IPA.

KAZMIRCZAK, Adria Mikoczak. Farmacêutica, especialista em Gestão Hospitalar, IAHCS. Pesquisadora convidada.

MASCARENHAS, Marcello Ávila. Orientador e Programa de Pós-Graduação em Biociências e Reabilitação do Centro Universitário Metodista do IPA. Rua Carlos Silveira Martins Pacheco n. 55 apto 802, bloco "i". Bairro Rio Branco, Porto Alegre, Rio Grande do Sul, Brasil. E-mail: mmasca2012@ gmail.com.

\section{RESUMO}

O objetivo deste estudo foi verificar a incidência de usuários após primeira internação com idade igual ou superior a 30 anos no Centro de Dependência Química do Hospital Parque Belém de Porto Alegre/RS e fazer uma reflexão sobre a sua reinserção à vida social. Adotou-se uma amostra composta de usuários de crack $(\mathrm{n}=81)$, fornecida através do banco de dados. Foi constatado que os usuários reinternados com 30 anos de idade ou mais são a minoria, 30,9\% dos casos; e 72\% deles obtiveram acesso ao tratamento hospitalar com ordem judicial. Esses usuários utilizavam o crack associado ao álcool (40\%) ou concomitante com outras drogas (44\%); revelou-se também que 16\% deles tinham alguma doença clínica associada. Com este estudo, observam-se as dificuldades de acesso à rede de atenção especializada e de qualidade, conforme garante a legislação brasileira.

Palavras-chave: Cocaína/crack; Dependência; Políticas públicas; Saúde pública.

\section{CRACK ADDICTS AND SOCIAL REINTEGRATION: REFLECTIONS ON A CASE STUDY}

\section{Abstract}

The aim of this study was to determine the incidence of 30 years old users, or upper, after a first hospital stay, in the Chemical Dependency Center of Parque Belém Hospital, in Porto Alegre / RS, and to reflect on their return to social life. We adopted a sample of crack users $(n=81)$ provided by the database. It has been found that readmitted users, with 30 years or more, are in the minority, $30.9 \%$ of cases; and $72 \%$ had access to hospital treatment by court order. These addicts used crack in combination with alcohol (40\%) or incombination with other drugs (44\%); it was also revealed that $16 \%$ had some associated clinical disease. With this study, we can observe the difficulties of gaining access to specialized care with quality, as guaranteed in the Brazilian legislation.

KeYwORDs: Cocaine/crack; Addiction; Public policies; Public health. 


\section{INTRODUÇão}

Organização Mundial da Saúde - OMS considera o uso abusivo de drogas como uma doença crônica e recorrente, constituindo um problema de saúde pública que vem ultrapassando todas as fronteiras sociais, emocionais e políticas, preocupando toda a sociedade (OMS, 2010; ANDRETTA \& OLIVEIRA, 2011). No mundo, estima-se que 14 milhões de pessoas realizam uso abusivo de cocaína e crack. No Brasil, de acordo com o II Levantamento Domiciliar sobre o uso de Drogas, realizado pelo Centro Brasileiro de Informações sobre Drogas Psicotrópicas - CEBRID, constatou-se que 7,2\% dos indivíduos do sexo masculino, entre 25 e 34 anos de idade, já usaram a droga, e dados epidemiológicos recentes mostram que o uso de cocaína/crack vem crescendo nos últimos anos entre os estudantes do ensino médio e fundamental, bem como entre os pacientes que procuram atendimento em clínicas especializadas (DALGALARRONDO, 2008; CEBRID, 2006; AGUILAR \& PILLON, 2005; LARANJEIRA et al., 2003).

Em virtude de ser um problema bastante complexo, no qual estão envolvidas várias dimensões, deve-se perceber a dependência química como sendo uma doença biopsicossocial. Em função disso, os modelos de tratamento necessitam de tipos de intervenções que incluam diversas estratégias de abordagem do problema, considerando elementos biológicos, psicológicos e sociais (SOUSA et.al., 2013). A dependência química é uma doença multicausal, que necessita de tratamento clínico e farmacológico e de intervenções com abordagem psicossocial, que contemplem as necessidades de saúde do usuário e de sua família. Essas iniciativas são essenciais para estabelecer condições que viabilizem o tratamento, a fim de estabilizar o quadro da dependência, garantir a remissão dos sintomas de abstinência e evitar a recaída. Assim, o tratamento e o plano de reabilitação psicossocial do dependente químico devem ser desenvolvidos por equipe multiprofissional e, nesta, os profissionais da saúde desempenham papel imprescindível no tratamento, reabilitação, reintegração social e atendimento familiar, colaborando ativamente nesse processo e oferecendo cuidado planejado, respeitando as especificidades de cada indivíduo (GUIMARÃES \& PAES, 2011; SILVA et.al., 2010; NETO \& ELKIS, 2007).

Nas últimas décadas, observou-se uma grande preocupação em estudar sobre os usuários de droga que buscam algum tipo de serviço para uma desintoxicação. Essa preocupação se deve à alta prevalência de internações, em torno de $46 \%$ dos usuários de crack, e à recaída após a alta hospitalar, trazendo problemas de níveis culturais, sociais e econômicos (GUIMARÃES et al., 2008; AGUILAR \& PILLON, 2005; FERREIRA FILHO et al., 2003).

Em alguns estudos que traçam o perfil do usuário de crack, nota-se um crescimento significativo de adultos usuários da referida droga, o que pode ser uma das hipóteses de uma possível falha no direcionamento das políticas públicas no que diz respeito à reinserção social, pois a política prescreve medidas de prevenção do uso e reinserção social; não ocorrendo isso, pode ocasionar a reincidência do uso e, por vezes, a criminalidade (GUIMARÃES et al., 2008; KESSLER, 2008; OLIVEIRA1 \& NAPPO, 2008; OLIVEIRA2 \& NAPPO, 2008; RAMOS, 2008; BRASIL, 2006; AGUILAR \& PILLON, 2005; CUNHA et al., 2004; FERREIRA FILHO et al., 2003; FERRI et al., 1997).

Observando a deficiência do sistema de saúde, em que esses usuários têm dificuldade de ingressar em ações qualificadas para a sua reinserção à vida social após a internação, diminuindo assim o número de reinternações e o impacto negativo à saúde pública, este trabalho visa verificar a incidência de usuários de crack em reinternação atendidos no Centro de Dependência Química do Hospital Parque Belém em Porto Alegre/RS, fazendo uma reflexão sobre reinserção social.

\section{Metodologia}

A presente pesquisa se constitui de um estudo transversal com uma abordagem híbrida com aspecto quantitativa, qualitativa, descritiva e exploratória. A 
análise quantitativa foi através da análise do banco de dados de pacientes usuários de crack em tratamento no Centro de Dependência Química - CDQUIM do Hospital Parque Belém, da cidade de Porto Alegre/ $\mathrm{RS}$, com idade igual ou superior a 30 anos em reinternação para desintoxicação. Para a estratégia metodológica qualitativa foram utilizados artigos científicos originais e de revisão voltados ao tema, através dos seguintes descritores em saúde: cocaínal crack, dependência, políticas públicas e saúde pública, pesquisados nas seguintes bases de dados: Scielo, Pubmed, Science Direct.

A coleta de dados foi precedida pela aprovação do estudo pelo Comitê de Ética emPesquisa, do Centro Universitário Metodista-IPA e autorização do Hospital Parque Belém, em que foi examinado o banco de dados $(\mathrm{n}=81)$ de pacientes do sexo masculino atendidos nesse centro, na segunda ou terceira reinternação, no primeiro semestre de 2010. Foram extraídos desse banco dados referentes à idade, se estavam na segunda ou terceira internação, se eram provenientes de ordem judicial, se possuíam doença clínica associada, se usavam o crack concomitantemente ao álcool ou outras drogas. Foram excluídos indivíduos que estavam na primeira internação e que apresentavam algum sintoma psicótico.

Para compilação e análise das variáveis qualitativas foi realizada uma análise descritiva, expressando em frequência. As análises foram realizadas com auxílio do programa Statistical Package for the Social Sciences-SPSS, versão 14.0.

\section{Resultados}

A amostra deste estudo foi composta por adultos do sexo masculino, em reinternação no Centro de Dependência Química do Hospital Parque Belém, de qualquer nível socioeconômico e com idade mínima de 18 anos e máxima de 53 anos, conforme banco de dados fornecidos por essa organização. O banco de dados possuía 97 cadastros de usuários internados, 81 deles em reinternação e apenas 25 indivíduos $(30,9 \%)$ com 30 anos de idade ou mais.

A Tabela 1 demonstra o perfil dos usuários de crack encaminhados por ordem judicial para que obtivessem um tratamento de desintoxicação, sendo a maioria deles (72\%) na segunda ou terceira internação e com 30 anos ou mais, tendo a idade máxima de 53 anos.

Podemos observar um maior número de usuários (48\%) de terceira internação que também fazem uso concomitante de álcool ou outra droga (maconha, ecstasy e/ou cocaína injetável), e 16\% dos usuários reinternados com mais de 30 anos têm algum tipo de doença clínica associada, como, por exemplo, hepatite C, diabetes, hipertensão arterial sistêmica, bronquite/ asma, vírus da imunodeficiência humana e/ou doenças cardíacas em geral.

Tabela 1 - Perfil dos usuários de crack $(\mathrm{N}=81)$ do Centro de Dependência Química do Hospital Parque Belém de Porto Alegre/RS no período de junho a agosto de 2010.

\begin{tabular}{|c|c|c|}
\hline Variável & $\begin{array}{c}\text { Reinternados } \\
(\mathrm{N}=56)\end{array}$ & $\begin{array}{c}\text { Reinternados } \\
\text { com } \geq 30 \text { anos } \\
(\mathrm{N}=25)\end{array}$ \\
\hline Idade mínima (anos) & 18 & 30 \\
\hline Idade máxima (anos) & 53 & 53 \\
\hline Ordem judicial (\%) & 61 & 72 \\
\hline Uso de crack (\%) & 58 & 56 \\
\hline Uso de crack e álcool (\%) & 38 & 40 \\
\hline Uso de crack e outras drogas (\%) & 41 & 44 \\
\hline Segunda reinternação (\%) & 59 & 52 \\
\hline Terceira reinternação (\%) & 41 & 48 \\
\hline Doença clínica associada (\%) & 12,5 & 16 \\
\hline
\end{tabular}

Fonte: Dados de pesquisa. 


\section{Discussão}

O estudo do perfil do usuário de drogas é de fundamental importância para a compreensão da natureza dos distúrbios decorrentes desse uso e para a formação de uma consciência crítica e direcionada a esse problema. Neste estudo foi constatado que, na maioria das vezes, os usuários na segunda internação, do sexo masculino, são jovens, com a faixa etária entre 18 e 29 anos (59\%), e é nessa faixa de idade que homicídios atingem o pico e avançam nas regiões metropolitanas. Já os usuários internados com 30 anos ou mais, na terceira internação, são a maioria, representando $48 \%$ da população estudada. Assim, observa-se que tanto adolescentes quanto adultos representam uma população exposta ao risco de morte por homicídios. Segundo Guimarães et al. (2008), o uso de crack propicia delitos, violência e aquisição de dívidas com o tráfico, deixando-os vulneráveis aos homicídios por vingança, participação em assaltos e/ ou queima de arquivo, pois muitos usuários encontram nessas alternativas a única maneira de tentar sustentar o vício que possuem.

No que se refere à internação por ordem judicial, a maioria (72\%) dos usuários com 30 anos ou mais teve acesso ao tratamento por esse meio. Aoferta de leitos psiquiátricos para dependentes químicos é apenas uma ação isolada, que pode até resolver questões pontuais, mas não a origem do problema. Deve-se considerar que uma gama de pacientes não se interna voluntariamente e muitos deles são encaminhados por familiares às emergências ou por ordem judicial e, portanto, precisam de um tratamento especializado, com profissionais e uma estrutura específica. Os serviços de saúde especializados devem ter, no seu quadro multiprofissional, pessoas com um poder de escuta, paciência e condições de não limitar suas formas de intervenção em ações técnicas (GUIMARÃES \& PAES, 2011; SILVA et.al., 2010; GUIMARÃES et al., 2008; NETO \& ELKIS, 2007; CUNHA et al., 2004).

Observou-se também que $40 \%$ dos usuários em reinternação, com idade igual ou superior a 30 anos, utilizam crack associado ao álcool e $44 \%$ deles consomem também outras drogas, como maconha, ecstasy e cocaína injetável. O uso combinado do crack com outras drogas possibilita o desenvolvimento da dependência física, química e psíquica, o que tende a dificultar a recuperação do usuário, retardando a sua reinserção social. A Organização Mundial de Saúde reconhece a dependência química como doença, porque há alteração da estrutura e no funcionamento normal da pessoa, não tendo causa única, mas sim uma série de fatores relacionados à saúde coletiva. Entretanto, devemos levar em conta que alguns fatores são mais predominantes nesse grupo de indivíduos expostos quando comparados com demais (OMS, 2010; OLIVEIRA1 \& NAPPO, 2008; OPAS, 2006; SANCHEZ \& NAPPO, 2002).

Quando não há um tratamento adequado, um atendimento integral, conforme preconizado na Lei Orgânica do SUS (BRASIL, 1990), dirigido aos usuários de drogas, a dependência química tende a prejudicar as habilidades sociais, como motivo de término de relacionamentos, lesões graves, hospitalizações e mortes violentas, agravando ainda mais o uso do álcool e outras drogas. Os transtornos mais comuns em comorbidade com os transtornos por uso de substâncias psicoativas são: depressão, transtornos de ansiedade, transtornos de conduta, transtorno de déficit de atenção e hiperatividade - TDAH e, numa extensão menor, a esquizofrenia e os transtornos da personalidade (SCHEFFER \& ALMEIDA, 2009; BARKIN, SMITH, DURANT, 2002).

Esta pesquisa revelou, também, que $16 \%$ desses usuários, com idade igual ou superior a 30 anos, têm alguma doença clínica associada, como a presença do vírus da imunodeficiência humana e da hepatite C. Neste momento o sujeito recuperado do uso de drogas se vê diante de um grande desafio que é o retorno ao meio familiar e social, necessitando um apoio assistencial a dependentes químicos (FERREIRA FILHO et al., 2003).

ALei 11.343/2006, que instituiu Sistema Nacional de Política Pública sobre Drogas - Sisnad, prescreve medidas de prevenção, atenção e reinserção social de usuários e dependentes, buscando uma harmonia entre 
as políticas de repressão ao tráfico, e dessa forma se inova quanto às medidas penais dispensadas para os usuários de drogas e o indivíduo que atua no tráfico das mesmas, representando um firme passo em direção ao desenvolvimento de uma abordagem (BACELLAR \& MASSA, 2010; BRASIL, 2006).

Para promover a inserção social através de ações intersetoriais, e regular a entrada na rede de assistência em saúde mental, através da atenção básica, foram criados no Brasil cerca de 690 Centros de Atenção Psicossocial - CAPS. O perfil populacional dos municípios é, sem dúvida, um dos principais critérios para o planejamento da rede de atenção à saúde mental nas cidades e para a implantação dos CAPS, e o critério populacional deve ser compreendido como um orientador para o planejamento. Tendo em vista que Porto Alegre tem uma população de $1.436 .123 \mathrm{em}$ 2009, deveríamos ter os cinco possíveis CAPS para $\mathrm{o}$ atendimento das pessoas portadoras de dificuldades mentais. No Rio Grande do Sul temos de 0,46 a 0,60 CAPS/100.000 habitantes, e a referência é de 1 CAPS para cada 100.000 habitantes (FIGUEIREDO, 2008; BRASIL, 2005). Praticamente a metade do considerado o ideal.

Os CAPS, dentro da atual política de saúde mental do Ministério da Saúde, são considerados dispositivos estratégicos para a organização da atenção em saúde mental. Eles devem ser territorializados, ou seja, devem estar dentro no espaço de convívio social dos que o utilizam e deve ser um serviço de resgate das potencialidades dos usuários (MINISTÉRIO DA SAÚDE, 2003). A reinserção social pode ocorrer a partir do CAPS, mas sempre direcionada à comunidade.

Diante desse problema, observamos a necessidade de haver uma maior intervenção por parte das políticas públicas na dependência química, não só nas drogas ditas ilícitas, mas principalmente em relação ao álcool, que, por ser uma droga lícita, está sendo consumido de forma excessiva e se tornando uma droga perigosa, pois traz consigo várias complicações clínicas, psicológicas, familiares e sociais.

Para que possamos ir à direção de uma resposta à sociedade sobre essa questão, precisamos de compromisso da parte dos gestores e população com a saúde pública na área de álcool e drogas e de um planejamento estratégico de prevenção no longo e curto prazos, frente à epidemia que estamos vivendo, pois, notavelmente, o principal objetivo da legislação é de evitar que o usuário seja confundido com um criminoso, e seja visto com um cidadão que necessita de um tratamento voltado à educação.

\section{Conclusão}

Este estudo demonstrou que a maioria da população usuária de crack, que está em sua segunda ou terceira internação no Centro de Dependência Química do Hospital Parque Belém de Porto Alegre/RS, é composta por indivíduos com idade igual ou superior a 18 anos e tem acesso ao serviço de desintoxicação através de ordem judicial, sendo composta de jovens em fase produtiva. O Sistema Único de Saúde tem se deparado com o aumento alarmante do número de usuários que procuram tratamento, exigindo uma rede estruturada de atenção psiquiátrica, com garantia de continuidade da assistência, sempre que necessário. A redefinição do modelo de atenção à saúde mental no país foi um avanço, no sentido de evitar que os doentes fossem excluídos da sociedade. No entanto, no Brasil ainda não existem serviços adequados e em quantidade compatível com a demanda.

Portanto, a ausência de suporte social, associado à estabilidade do núcleo familiar e disponibilidade de rede de tratamento adequado, têm agravado a situação do uso de crack. Logo, considera-se que, além de constituir uma estimativa importante do perfil da população estudada, esta pesquisa sugere desenvolver estudos que acompanhem os usuários das unidades de desintoxicação após sua alta hospitalar, a fim de planejar, quantificar e, principalmente, qualificar as ações que são prestadas a eles pelos serviços públicos de saúde no Brasil.

Além de programas educativos que fazem a prevenção, são necessárias ações conjuntas entre os setores, em todos os âmbitos, para que se inclua a atenção básica na vida dessa população, enfatizando 
a promoção e educação em saúde, a prevenção de doenças e melhoria da qualidade de vida, mudando a relação e o modo de viver entre os indivíduos usuários de crack e demais pessoas vulneráveis ao consumo de drogas.

\section{REFERÊNCIAS}

AGUILAR, L. R.; PILLON, S. C.. Percepción de tentaciones de uso de drogas en personas que reciben tratamiento. Revista Latino-Americana de. Enfermagem. n. especial, p. 790-7, set/out. 2005.Acesso em: 26 jun. 2010.

ALMEIDA FILHO, N. O conceito de saúde e a vigilância sanitária: Notas para a compreensão de um conjunto Organizado de práticas de saúde. Documento comissionado pela ANVISA para discussão no I Seminário Temático Permanente da Agência Nacional de Vigilância Sanitária, Brasília, DF, 18 de out. 2000. Disponível em: <http:/ /www.anvisa.gov.br/institucional/snvs/coprh/ relatorios/gestao_2000_2002/

plano_trabalho_anexosi.htm/conceito.pdf

\section{ANDRETTA, L.\& OLIVEIRA, M.S. A entrevista} motivacional em adolescentes usuários de droga que cometeram ato infracional. Psicologia: Reflexão e Crítica, 24(2), 218-226. 2011.

\section{BACELLAR, R. P.; MASSA, A. A. G. Programa} de Alternativas Penais em Prevenção do Uso de Drogas - PAPPUD. Paraná. Disponível em: <http:// portal.tjpr.jus.br/download/je/projetos/ abordagem_juiz.pdf $>$. Acesso em: 18 jun. 2010.

BARKIN, S. L.; SMITH K,. S.; DURANT, R. H. Social skills and attitudes associated with substance use behavior among young adolescents. Journal of Adolescent Health. v. 30, p.448-454, 2002.

\section{BELISÁRIO, S. A. Associativismo em Saúde}

Coletiva: um estudo da Associação Brasileira de
Pós-graduação em Saúde Coletiva - ABRASCO.

Campinas, [s.n.], 2002.

BRASIL. Lei no 8.080 de 19 de setembro de 1990. Dispões sobre as condições para a promoção, proteção e recuperação da saúde, a organização e o funcionamento dos serviços correspondentes, e dá outras providências. Diário Oficial da União, Brasília, DF, 20 set. 1990. p.18055.

BRASIL. Lei no ${ }^{\circ} 11.343$ de 23 de agosto de 2006. Institui o Sistema Nacional de Políticas Públicas sobre Drogas - SISNAD; prescreve medidas para prevenção do uso indevido, atenção e reinserção social de usuários e dependentes de drogas; estabelece normas para repressão à produção não autorizada e ao tráfico ilícito de drogas; define crimes e dá outras providências. Diário Oficial da União, Brasília, DF, 24 ago. 2006.

BRASIL. Ministério da Saúde. Secretaria de Atenção à Saúde. DAPE. Coordenação Geral de Saúde Mental. Reforma Psiquiátrica e Política de Saúde Mental no Brasil. Documento apresentado à Conferência Regional de Reforma dos Serviços de Saúde Mental: 15 anos depois de Caracas. OPAS. Brasília, nov. 2005.

CEBRID. II Levantamento domiciliar sobre o uso de drogas psicotrópicas no Brasil: estudo envolvendo as 108 maiores cidades do país - 2005. São Paulo: CEBRID. p.33, 2006.

\section{CONFERÊNCIA INTERNACIONAL SOBRE} CUIDADOS PRIMÁRIOS DE SAÚDE.

Declaração de Alma-A ta. Cazaquistão, 6-12 set. 1978. Disponível em: <http://www.opas.org.br/ promocao/uploadArq/Alma-Ata.pdf $>$ Acesso em: 19 ago. 2010.

CORDEIRO, D. C.; FIGLIE, N. B.; LARANJEIRA, R. R. Boas práticas no tratamento do uso de dependência de 
substâncias. São Paulo: Rocca, 2007.

CUNHA, P. J.; NICASTRI, S.; GOMES, L. P.; MOINO, R. M.; PELUSO, M. A. Alterações neuropsicológicas em dependentes de cocaína/crack internados: dados preliminares. Revista Brasileira de Psiquiatria. n. 26, p. 103-6, 2004.

DALGALARRONDO, P.. Psicopatologia e semiologia dos transtornos mentais. 2 ed. Porto Alegre: Artmed, 2008.

ELSEN I. Cuidado familial: uma proposta inicial de sistematização conceitual. In: ELSEN I, MARCON, S. S, SILVA, M.R.S. (Org.) O viver em família e sua interface com a saúde e a doença. 2. ed. Maringá: Eduem, p.398, 2004.

FERREIRAFILHO, O. F.; TURCHI, M. D.; LARANJEIRA, R. R.; CASTELO, A. Perfil sociodemográfico e de padrões de uso entre dependentes de cocaína hospitalizados. Revista de Saúde Pública. n. 37, p. 751-9, 2003.

FERRI, C. P.; LARANJEIRA, R. R.; SILVEIRA, D. X.; DUNN, J.; FORMIGONI, M.L. O. S.

Aumento da procura de tratamento por usuários de crack em dois ambulatórios na cidade de São Paulo, nos anos de 1990 a 1993. Revista da Associação Médica Brasileira. n. 43, p. 25-8, 1997.

FIGUEIREDO, N. M. A. Ensinando a cuidar em Saúde Pública. 2 ed. São Caetano do Sul,: Yendis Editora, 2008.

GUIMARÃES, C. F.; SANTOS, D. V. V.; FREITAS, R. C.; ARAÚJO, R. B. Perfil do usuário de crack e fatores relacionados à criminalidade em unidade de internação para desintoxicação no Hospital Psiquiátrico São Pedro de Porto Alegre (RS). Revista de Psiquiatria do Rio Grande do Sul. n. 30; p. 101-8, 2008.
GUIMARÃES, A. N.; PAES, M. R. Causas de recaída e de busca por tratamento referidas por dependentes químicos em uma unidade de reabilitação. Colombia Médica, v. 42, n. 2 Supl 1, 2011.

IBGE. Instituto Brasileiro de Geografia e Estatística. Coordenação de População e Indicadores Sociais. Estimativas das Populações Residentes em $1^{\circ}$ de julho de 2009, segundo os municípios. Disponível em: <http://www.ibge.gov.br/home/ estatistica/populacao/estimativa2009/ POP2009_DOU.pdf> Acesso em: 11 ago. 2010.

KESSLER, F.; PECHANSKY, F.. Uma visão psiquiátrica sobre o fenômeno do crack na atualidade. Revista de Psiquiatria do Rio Grande do Sul. n. 30, p. 96-98, 2008.

LARANJEIRA, R. R.. OLIVEIRA, R. A.; NOBRE, M. R. C.; BERNARDO, W. M. Usuários de substâncias psicoativas: abordagem, diagnóstico e tratamento. 2. ed. São Paulo: Conselho Regional de Medicina do Estado de São Paulo/Associação Médica Brasileira, 2003.

NETO, L.M.R., ELKIS H. Psiquiatria básica. 2. ed. Porto Alegre: Artmed; 2007.

MEDINA, N.A.; FERRIANI, M.G.C. Protective factors for preventing the use of drugs in the families of a Colombia locality. Rev. Latino-Am.

Enfermagem, v.18, p. 504-12, 2010.

MIRANZI, M. A. S.; ASSIS, D. C.; RESENDE, D. V.; IWAMOTO, H. H.. Compreendendo a história da saúde pública de 1870-1990. Saúde Coletiva. n. 41, p. 157-162, 2010.

MINISTÉRIO DA SAÚDE. Coordenação de 
Saúde Mental. Coordenação de Gestão da Atenção Básica. Saúde mental e atenção básica: o vínculo e o diálogo necessários. n. 1, p.2-3, 2003.

OLIVEIRA1, L. G.; NAPPO, S. A.. Caracterização da cultura de crack na cidade de São Paulo: padrão de uso controlado. Saúde Pública. n. 42, p. 664671, 2008.

OLIVEIRA2, L. G.; NAPPO, S. A.. Crack na cidade de São Paulo: acessibilidade, estratégias de mercado e formas de uso. Psiquiatria Clínica. n. 35, p. 212-8, 2008.

OPAS, Organização Pan-Americana de Saúde. Guia para atenção e manejo integral de usuários de drogas vivendo com HIV/AIDS na América Latina e Caribe. Washington, D.C,: OPAS, 2006.

OEDT, Observatório Europeu da Droga e da Toxicodependência. Evolução recente das políticas e da legislação no domínio da droga. Relatório Anual, Lisboa, 2005. Disponível: <http:// www.abead.com.br/artigos/arquivos/evolucao.pdf $>$. Acesso em: 07 mar. 2010.

OMS, ORGANIZAÇÃO MUNDIAL DA SAÚDE. Classificação Internacional de Doenças. 9 ed. São Paulo: OMS/OPS, 2010.

PAIM, J. S.; ALMEIDA FILHO, N. Saúde coletiva: uma "nova saúde pública" ou campo aberto a novos paradigmas. Saúde Pública. n. 32, p.299-316, 1998.
RAMOS, S. P.. O crack, o pai e os psiquiatras e psicanalistas. Psiquiatria do Rio Grande do Sul.n. 30, p. 99-100, 2008.

SANCHEZ, Z. M.; NAPPO, S. A.. Sequência de drogas consumidas por usuários de crack e fatores interferentes. Saúde Pública. n. 36, p.420-30, 2002.

SCHEFFER, M.; PASA, G. G.; ALMEIDA, R. M. $M$. Atenção, ansiedade e raiva em dependentes químicos. Psicologia da PUCRS, v.40, n.2, p.235244, 2009.

SCHENKER, M; MINAYO M.C.S. Fatores de risco e de proteção para o uso de drogas na adolescência. Ciências de Saúde Coletiva. v.10, n.3, p.707-17, 2005.

SELEGHIM, M. R. et al. Vínculo familiar de usuários de crack atendidos em uma unidade de emergência psiquiátrica. Rev. Latino-Am. Enfermagem, Ribeirão Preto, v. 19, n. 5, out. 2011.

SILVA L.H.P., BORBAL.O., PAES M.R., Guimarães AN, Mantovani MF, Maftum MA. Perfil dos dependentes químicos atendidos em uma unidade de reabilitação de um hospital psiquiátrico. Rev. Esc Anna Nery - Rev Enferm.; v.14, p.58590, 2010.

SOUSA, Patrícia Fonseca et al . Dependentes químicos em tratamento: um estudo sobre a motivação para mudança. Temas psicol., Ribeirão Preto, v. 21, n. 1, jun. 2013.

RECEBIDO EM 30/3/2014

ACEITO EM 30/9/2014 